\title{
Social Media: Institutionalisierungsprozesse als interdisziplinäre Forschungsaufgabe
}

\author{
Plattformökonomie und die Macht der neuen Intermediäre als Herausforderung \\ für die Sozialwissenschaften
}

Die MedienWirtschaft öffnet sich für interdisziplinär angelegte Beiträge, um die medienwirtschaftlich relevanten «Perspektiven der digitalen Transformation» angemessen adressieren zu können (vgl. Gläser et al. 2018: S. 4 f.). Diese Öffnung erscheint als sinnvoll, ja als notwendig. Aus gesellschaftlicher und somit auch aus genereller sozialwissenschaftlicher Perspektive ist die digitale Transformation dabei vor allem deshalb relevant, da die traditionellen Massenmedien als herkömmliche gesellschaftliche Intermediäre einem De-Institutionalisierungsprozess unterliegen, während sich wiederum die sozialen Medien offenbar in einem herausforderungsreichen Prozess einer gesellschaftlich relevanten Neu-Institutionalisierung befinden. Die fundierte Untersuchung der Chancen und Risiken dieses Institutionalisierungsprozesses der neuen Intermediäre ist lediglich interdisziplinär zu leisten. Da dieser Prozess von mächtigen kapitalistisch-privatwirtschaftlich konstituierten Plattform-Konzernen vorgeprägt wird, die die neuen digitalen Intermediäre betreiben, ist eine Renaissance der um die Jahrtausendwende von Marie Luise Kiefer (vgl. Kiefer 2001) konzipierten institutionenökonomisch orien- tierten Medienökonomik als interdisziplinäres Bindeglied zwischen den Wirtschafts- und Kommunikationswissenschaften wünschenswert. Im Hinblick auf die analytische Beschreibung und Erklärung kommunikativ bzw. diskursiv fundierter Formen der Institutionalisierung sind indes auch institutionenökonomische Ansätze augenfällig nicht geeignet. Dies ist die Domäne der Kommunikationswissenschaft in der interdisziplinären Aufgabenteilung. Der Beitrag zeigt mögliche Ansätze und Aspekte sowohl der interdisziplinären wie auch der kommunikationswissenschaftlichen Untersuchung der skizzierten De- und NeuInstitutionalisierungsprozesse auf.

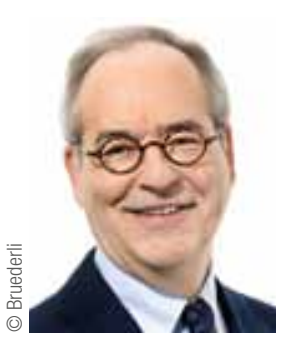

\section{Prof. em. Dr. Otfried Jarren}

Universität Zürich, Institut für Kommunikationswissenschaft und Medienforschung, Prof. mit besonderen Aufgaben

o.jarren@ikmz.uzh.ch

Schlüsselbegriffe: Soziale Medien I Plattformen I Intermediäre I Institutionentheorie I Institutionalistische Analysen

Für die gesellschaftliche Kommunikation sind die Massenmedien, also Presse, Radio und Fernsehen, noch immer relevant. Sie erzeugen und bündeln journalistische Produkte und koppeln diese mit anderen informierenden, unterhaltenden und werbenden Angeboten zu einer integralen publizistischen Leistung. Diese Leistungen dienen der Selbstbeobachtung wie der Selbstverständigung der Gesellschaft. Nur die Massenmedien als gesellschaftliche Institutionen stellen den Journalismus auf Dauer, garantieren publizistische Leistungen in einer spezifischen Qualität, und nur sie streben mit inrem Angebot gesamtgesell- schaftliche Relevanz an. Die Massenmedien verstehen sich als Intermediäre, und sie nehmen eine öffentliche Aufgabe wahr, die innen auch rechtlich zugewiesen wird (vgl. Donges 2006; Jarren 2008, 2018a). Im Zusammenspiel mit den Akteuren der Gesellschaft und in ihrem Zusammenwirken miteinander ermöglichen sie Koorientierung. Sie stellen die allgemeine Öffentlichkeit her, die insbesondere für alle gesamtgesellschaftlichen Entscheidungsprozesse, vorrangig die politischen Entscheidungen, von herausragender Bedeutung sind. 
Trotz dieser Leistung büßen sie jedoch ihre zentrale wie dominante institutionelle Position in der gesamtgesellschaftlichen Information wie Kommunikation ein: Sie verlieren - zumindest in Publikumssegmenten - an Vertrauen wie Glaubwürdigkeit, vor allem aber verlieren sie an Reichweite; sie durchdringen die Gesamtgesellschaft immer weniger. Damit - und das kann man derzeit empirisch beobachten - verlieren sie aber auch ihre institutionelle Position als gesellschaftliche Zentralakteure im Vermittlungssystem, die zuvor über Jahrzehnte hinweg für die Herstellung von Öffentlichkeit wie für die Institutionalisierung der Öffentlichen Meinung sorgten. Im Zuge des Institutionalisierungsprozesses von neuen Intermediären, vor allem von Social Media-Plattformen, büßen sie darüber hinaus möglicherweise aber sogar ihre institutionelle Legitimität ein.

Es gilt deshalb, die Intermediäre als Institutionen wie die institutionellen Prozesse, die sie ermöglichen, zu analysieren. Die derzeit stattfindenden Institutionalisierungs- wie De- oder Re-Institutionalisierungsprozesse sollten von den Sozialwissenschaften, insbesondere der Kommunikationsund Wirtschaftswissenschaft, in enger Kooperation mit der Rechtswissenschaft, theoretisch gefasst wie empirisch kooperativ bearbeitet werden (vgl. Jarren 2016). Im vorliegenden Beitrag wird auf Social Media-Plattformen fokussiert: Sie werden als die institutionellen Herausforderer der Massenmedien und des von inm institutionalisierten Journalismus aufgefasst. Es wird dargelegt, welche Problemstellungen und Themen ein institutionentheoretisch inspiriertes interdisziplinäres Forschungsprogramm zur Beschreibung wie zur Erklärung der hier postulierten Neu- wie De-oder Re-Institutionalisierungsprozesse aufgreifen könnte.

\section{Die Krise von Massenmedien und Journalismus}

Gebündelte publizistische Angebote von Zeitungen oder linear verbreitete Radio- und Fernsehprogramme verlieren an Rezipienten, sie verlieren an gesamtgesellschaftlicher Reichweite, und sie können im Markt (Nutzer- wie Werbemarkt) ihre Preise nicht mehr durchsetzen - mit Folgen für die Finanzierung journalistischer Leistungen. Die Abonnementsbereitschaft geht zurück, die Legitimität für Gebühren bzw. Abgaben (öffentlich-rechtlicher Rundfunk) sinkt, Bezahlschranken im Onlinebereich sind kaum einträglich durchsetzbar, Preismodelle für allgemeine journalistische Leistungen sind nicht existent (vgl. Lobigs 2017, 2018a). Der individuelle wie gesellschaftliche Wert von publizistischen Angeboten, insbesondere aber von konkreten journalistischen Leistungen, ist nicht sozial definiert und gesellschaftlich zudem nicht verankert. Journalistische wie publizistische Qualitäten gelten gemeinhin als unklar, nicht definierbar, nicht sicher ermittel- wie bewertbar - allen Qualitäts(messungs)initiativen zum Trotz. Das nicht vorhandene Wert- wie Qualitätsverständnis, das bereits in der
Branche selbst auffällig ist und sich bspw. am Nichtvorhandensein standardisierter Qualitätssicherungssysteme zeigt, wird im Kontext der Institutionalisierung insbesondere von Social Media-Plattformen zu einem Problem: Die Leistungen der Massenmedien erweisen sich - trotz allen Betonungen von der Relevanz der „Marken“ - insgesamt als unklar. Es ist wohl so: Massenmedien stammen aus dem industriellen Zeitalter. Sie passen sozio-kulturell nicht mehr in die moderne Dienstleistungsgesellschaft, in der auf Kollaboration zwischen Produzenten und Konsumenten gesetzt wird und sich der Wert einer Leistung aus dem Zusammenwirken von Produzenten und Konsumenten ergibt (vgl. zu dieser Idee Kiefer 2017).

Das publizistische Her- wie Bereitstellungsprinzip wie die journalistische Leistung der Massenmedien selbst verlieren also an gesamtgesellschaftlicher Relevanz, weil es nun, mit dem Aufkommen neuer Intermediäre, noch weitere Unsicherheiten bezüglich der Wertdefinition wie der Leistungsbeurteilung gibt. ${ }^{1}$ Zudem werden neue Erwartungen an die Massenmedien herangetragen, die sie nicht (so vor allem gegenüber jungen Nutzern) oder nur noch partiell (so gegenüber ältere Nutzern) erfüllen können. ${ }^{2}$ Aufgrund der neuen Vermittlungsinstitutionen wie -möglichkeiten können die bisherigen publizistischen Leistungen von den Nutzenden neu betrachtet werden.

\section{Neue mediale Vermittler und eine neue Vermittlungsstruktur}

Bezüglich Information und Kommunikation haben sich mit den Social Media neue Intermediäre mit anderen Bereitstellungsformen, Auswahlformaten wie Inklusions- und Beteiligungsmöglichkeiten in sehr kurzer Zeit etabliert. Sie sind neu, anders, innovativ. Sie basieren auf anderen Geschäftsmodellen (kostenlos, allgemeine Zugänglichkeit, globale Verbreitung, Real-Time-Nutzertargeting in der Werbung). Sie sprechen mit ihren Personalisierungs- und Konfektionsangeboten Individuen gezielt an. Sie ermöglichen Individuen vielfältige Formen an Kollaboration. Aber diese neuen Anbieter sind nicht zu den Massenmedien lediglich hinzugetreten, also nicht Teil der etablierten Medienbranche mit ihren Normen und professionellen (zumal journalistischen) Standards geworden. Sie setzen sich von ihnen hingegen gezielt ab.

\footnotetext{
1 Das Bewertungsproblem ist dabei auch auf Ausdifferenzierungsprozesse des traditionellen Mediensystems zurückführen. Im Unterschied zur kommunikationswissenschaftlichen Annahme, dass es "den" Journalismus (Singular) gibt, gibt es eine Vielzahl von „Journalismen“. Längst übertrifft die Anzahl der in spezifischen Verbänden sogar organisierten „Fachjournalisten“ die Zahl an Journalisten, die bei den allgemeinen Massenmedien (universelles, aktuelles Angebot) arbeiten.

2 Nur unter Monopolbedingungen konnten die Massenmedien auf publizistische Differenzierung verzichten. Die Tagespresse hatte schon länger den Spagat zwischen „Jung" und „Alt“ zu meistern; auf eine Produktdifferenzierung wurde verzichtet.
} 
Bei diesen Marktzutritten handelt es sich nicht - wie vielfach noch immer im wissenschaftlichen wie (medien-)politischen Diskurs oder bei Regulierungsüberlegungen angenommen wird - um Konvergenz, also um einen Prozess an Kooperation wie Konkurrenz zwischen alten und neuen Marktanteilnehmern in einem bestehenden (gleichen) Markt und unter gleichen berufsprofessionellen Bedingungen (Journalismus), sondern um institutionelle ${ }^{3}$ Neuzutritte: Die neuen Intermediäre sind neue Institutionen, sie lösen deshalb einen fundamentalen institutionellen Wandel aus (vgl. Gillespie 2018). Die Gesellschaft ist durch den Zutritt dieser neuen Intermediäre mit einem massiven sozio-technischen, sozio-kulturellen, sozio-ökonomischen und auch sozio-politischen Wandel konfrontiert, der neue Institutionen für die gesellschaftliche Vermittlung etabliert, die deutlich über das Leistungsspektrum der Massenmedien hinausweisen (Transformation). Social Media-Plattformen, verbunden mit künstlicher Intelligenz (Algorithmen, Big Data, Deep Learning), haben disruptive Folgen für die Massenmedien und deren bislang vorherrschendes gesellschaftliches Vermittlungsmonopol. ${ }^{4}$

Social Media sind nicht nur neue Vermittlungsinstitutionen, sondern sie etablieren zudem auch eine neue gesellschaftliche Vermittlungsstruktur. Die neuen Social Media-Plattformen sind keine klassischen, d. h. publizistischen, Medien. Sie ermöglichen
- Einzelnen,
- Gruppen,
- Netzwerken oder
- Organisationen

die Beschaffung und Verbreitung von Informationen jedweder Art. Und sie dienen dazu, sich zu organisieren - punktuell wie dauerhaft Netzwerke auszubilden (Netzwerkgesell-

3 "Institutionalisierung findet statt, sobald habitualisierte Handlungen durch Typen von Handelnden reziprok typisiert werden" (Berger/Luckmann 2007: 58). Durch wiederkehrende Interaktionen findet eine Stereotypisierung von Akteuren, Rollen und Erwartungen statt, die zu gewissen Formen des Vertrauens führen. Es bilden sich Regeln und Normen aus. Institutionen können durch rechtliche wie normative Vorgaben gebildet werden, sie können aber auch - wie im Falle der Institutionalisierung von Social Media-Plattformen angenommen wird - die Konsequenz nicht intendierter Effekte von Handlungen sein. Bei Social Media ist ein solcher Prozess auch deshalb wahrscheinlich, weil sich in sehr kurzer Zeit sehr viele Individuen beteiligt haben: man folgte den Bekannten. „Follower“ zu haben war bzw. ist sogar ein soziales Reputationskonzept einiger Social Media-Plattformen. Bestehende individuelle Unsicherheiten konnten durch die öffentliche Sichtbarkeit der Plattform, aufgrund der Beteiligung von öffentlichen Personen wie zugleich auch aus dem eigenen Bekanntenkreis, reduziert werden. Rasche Reichweite, damit steigender individueller Nützlichkeitsgrad und Follower als soziale Währung sind Begründungen für die zügige Institutionalisierung. Ferner haben die Plattformbetreiber durch Formen des „institutional work", vor allem vermittels Kommunikation (Leitideen, Werbung, PR etc.), dazu beigetragen, gänzlich neue Kommunikationsmöglichkeiten institutionell zu vermitteln. Vgl. zum Institutionenbegriff wie -konzept auch die Darstellung in Katzenbach (2018)

4 Von den disruptiven Folgen sind auch andere Akteure, die auf Austauschbeziehungen wie Vermittlungsleistungen mit der Gesamtgesellschaft angewiesen sind, betroffen - so Parteien wie aber auch Regierungen oder Verwaltungseinheiten sowie nicht zuletzt auch Unternehmen in vielen Wirtschaftsbranchen. schaft). Ihre Etablierung hat vielfältige Folgen, so für das institutionelle und somit auch das (verfassungs-)rechtliche Medienverständnis: Mit inrem Marktzutritt ist der nationalstaatlichen Regulierung der tradierte Medienbegriff (so der Rundfunkbegriff) der Gesellschaft sowie das bislang geltende Verständnis von gesellschaftlicher Vermittlung über „die Medien“ (Akteure, Normen, Regeln) abhandengekommen. Die bestehende korporatistisch verfasste und von den machtvollen kollektiven Interessenorganisationen bestimmte gesellschaftliche Vermittlungsstruktur mit ihren als legitim angesehenen Massenmedien und Vermittlungsformen im Zentrum ist ins Wanken geraten (vgl. Jarren 2018b). Deshalb erleiden auch die öffentlich finanzierten Massenmedien Reichweiten- wie Anerkennungsverluste, obwohl sie mindestens in Deutschland bisher keine ökonomischen Einbußen zu verzeichnen haben.

Hat der Prozess der Institutionalisierung neuer Intermediäre einen De-Institutionalisierungsprozess für die Massenmedien zur Folge? Sichtbar ist derzeit, dass sich die gesellschaftlichen Vermittlungsformen wie -strukturen verändert haben. Die neuen Strukturen sind aber noch nicht vollends erkennbar. Und für eine neue Struktur hat die Gesellschaft weder Leitbilder noch Normen bislang entwickelt, geschweige denn Gesetze. Aber die neuen Anbieter wissen, was sie wollen. Und sie haben im globalen Medien- und Kommunikationsmarkt Erfolg.

Die neuen Intermediäre in Form der Social Media ermöglichen, und darin liegt wohl ihre Bedeutung wie inr institutionelles Potential, eine Steigerung der gesellschaftlichen Vermittlungsmöglichkeiten, und zwar sowohl für Individuen, Gruppen, Netzwerke und Organisationen wie die Gesamtgesellschaft. Vor allem Individuen - und das ist im Kern die institutionelle Innovation - verfügen über erheblich ausgeweitete Informations-, Kommunikations- und (Selbst-) Organisationsmöglichkeiten über Medien. Und diese Medien (so in Form des Smartphones) führen die Individuen stets mit sich. Alle Nutzer sind ständig online und partiell auch stets aktiv (vgl. Vorderer et al. 2015). Diese Medien sind persönliche Medien, die als Besorgungsdienstleister fungieren. ${ }^{5}$ Mittels dieser Geräte wird Alltag organisiert. Sie sind keine vom Individuum räumlich wie sozial entfernt wirkenden Apparate, sondern persönliche Hilfsmittel. Herkömmliche technische wie soziale Flaschenhälse, um andere zu erreichen wie zu interagieren, sind entfallen. Mit den neuen Intermediären können in steigendem Maß Daten beschafft wie verteilt werden. Sie unterstützen damit die

\footnotetext{
5 Besorgung und Besorgungsdienstleister wird hier in einem allgemeinen Sinne verstanden (vgl. dazu Schrader 2018). Das Smartphone dient als persönlicher technischer Assistent für vielfältige Aufgaben der Lebensgestaltung, so bezüglich der Selbstorganisation bzw. des Selbstmanagements (von der Terminverwaltung bis hin zur Routenplanung) wie der Organisation der Beziehungen zu Dritten (im privaten wie im geschäftlichen Kontext). Besorgung verweist hier also auf die aktive Komponente durch die Nutzenden.
} 
mobile, auf ständigen Austausch wie Interaktion angewiesene Gesellschaft. Statt von Medien kann man von neuen Intermediären oder Plattformen sprechen. ${ }^{6}$

\section{Plattformen als neue Vermittlungsakteure}

Plattformen sind auf zwei- oder mehrseitigen Märkten tätig, und sie bringen (Kunden-) Gruppen mit komplementären Bedürfnissen zusammen (vgl. grundlegend Evans/Schmalensee 2016). Bezeichnet werden damit (im Medien- bzw. Kommunikationssektor) so unterschiedliche Anbieter wie Suchmaschinen, Soziale Netzwerke, App-Plattformen, User-Generated-Content-Plattformen, (Micro-)Blogging-Plattformen, News-Aggregatoren oder Verkaufsplattformen (vgl. Schulz/ Dankert 2016). Hier wird auf jene Intermediäre fokussiert, die für die Beschaffung, Aggregation, Selektion, Präsentation, Weiterleitung oder Bewertung von Informationen wie Wissen oder für Kommunikationszwecke genutzt werden.

Massenmedien sind seit der Etablierung der neuen Intermediäre nicht mehr exklusive Vermittler von Information wie Werbung. Selbst auf einer Verkaufsplattform kann man Informationen, Werbung oder PR-Beiträge nutzen und Transaktionen unmittelbar auslösen. ${ }^{7}$ Verkaufsplattformen bieten aber auch Unterhaltung wie Informationen an, um Nutzer auf die Plattform zu locken. Plattformen aller Art können deshalb potentiell die Leistungen von Massenmedien wie des Journalismus (teilweise) übernehmen oder simulieren („Pseudojournalismus“). Vielfach erwecken sie den Eindruck, sie seien wie Medien und Journalismus für die Nutzerinnen und Nutzer tätig, so indem sie Informationen von (bekannten) Medien distribuieren. Vielfach sind auch Journalistinnen und Journalisten auf Blogs oder Social Media als Personen präsent.

Das Machtpotential von Plattformen, der Plattformökonomie, ist offensichtlich. Dies auch deshalb, weil sie eine große Zahl

6 Streaminganbieter, so wie Netflix, werden hier nicht näher betrachtet. Ebenso werden durch Plattformen institutionalisierte Marktplätze nicht weiter beachtet (bspw. Amazon), obwohl auch diese - vielfach zumeist vorrangig unterhaltende Angebote machen. Von beiden Typen von Intermediären gehen zumindest indirekte Effekte auf die klassischen Massenmedien aus: Verluste an Aufmerksamkeit, Ressourcenverluste etc.

7 Beispielweise „Prime" von Amazon: Auf „Prime“ werden zahlreiche eigene Angebote gebündelt: „Prime Music“, „Prime Video“, „Prime Photos“ etc., aber auch Angebote Dritter. Damit wird auf „Prime“ ein allgemeines Angebot ebenso ermöglicht wie ein Shop-in-Shop-Konzept. Der Kundennutzen (aus einer Hand ein großes Angebot, schnell, bequem, günstig) steht im Mittelpunkt. Durch die Angebotsbreite soll der Nutzerwert steigen und die Bindung der Kunden erhöht werden. Dazu trägt ein zentrales Login mit individuellen Nutzerkonten bei. Diese ermöglichen Personalisierungs- wie Konfektionalisierungsmöglichkeiten. Die traditionellen Massenmedien realisieren das Gegenteil dieses Konzepts: Sie bieten nicht die quantitativ oder qualitativ besseren Leistungen anderer - so bspw. der spezialisierten Medien - an, sondern produzieren auch in solchen Feldern, in denen sie allenfalls Randkompetenzen haben (so in der Sportberichterstattung). Zugleich aber zwingen sie ihre Nutzer dazu, auch diese Leistungen mit zu erwerben. Mit überhöhten normativen Ansprüchen, im Sinne von „Alles für Alle“, werden diese Angebote legitimiert. Beim öffentlich-rechtlichen Rundfunk wird für diese Bereitstellung der "Integrationsauftrag" legitimatorisch bemüht. an Teilnehmenden erreichen. Neben der großen Reichweite kommt hinzu, dass sie zugleich spezifische Gruppen wie Einzelne erreichen (vgl. zur Wertschöpfung in Datenmärkten Bründ//Matt/Hess 2016). Diese umfassende Reichweite zu erzielen ist nicht allen, aber einer Gruppe an global agierenden Unternehmen, so Google oder Facebook, in relativ kurzer Zeit gelungen. Dabei ist das Prinzip Reichweite nicht neu: Reichweite ist ein institutionelles Grundprinzip, so auch der Massenmedien. Sie streben große Reichweiten an, was ihnen aber immer weniger gelingt. Und sie können ihr Angebot zudem nur begrenzt differenzieren, selektiv verteilen oder personalisieren. Sie können aufgrund ihrer industriellen Produktions- wie Distributionsweise keine unmittelbare Beteiligung ermöglichen. Von der Organisationsverfassung sind sie zentralistisch wie hierarchisch bestimmt. Sie bieten ihren Nutzern nur im begrenzten Maß Möglichkeiten für Rückmeldungen. Vor allem aber verfügen die Massenmedienunternehmen nur über geringe Informationen über ihre Nutzer, so um gezielt in den Werbe-, Publikums- wie Nutzermärkten mit (personalisierten) Angeboten agieren zu können.

Die neuen Plattformen beeinflussen

- das Geschäftsmodell der Massenmedienbranche,

- das institutionelle Grundverständnis der Massenmedien und des Journalismus wie

- die organisationale Verfasstheit von und die Arbeitsweise in Medienorganisationen

und dadurch die Finanzierung des Journalismus. Mehr als das: Sie stellen die Institution Massenmedium mit ihrem spezifischen Leistungsspektrum in Frage. ${ }^{8}$

\section{Spannungsreicher wie widersprüchlicher Institutionalisierungsprozess}

Standen am Anfang der Etablierung des Internets, insbesondere der Social Media, hohe demokratische Erwartungen ${ }^{9}$, so die nach mehr Partizipation, macht sich nun Ernüchterung breit: Manipulation und unerlaubte Weitergabe von Daten, Datenunsicherheit, Identitätsdiebstahl, Cyber Attacks, Filter Bubble, Echo Chambers, Social Bots, die mögliche Manipulation von Öffentlicher Meinung wie Wahlen durch Trolle wie Algorithmen. ${ }^{10}$ Das Wissen um manipulierte Suchergebnisse oder von Manipulationen durch Social Bots auf Social Media-Plattformen lösten und lösen Irritationen aus. Doch markante Verhaltensänderungen sind nicht, zumindest

8 Es stellt sich generell die Frage: Hatte Journalismus überhaupt ein eigenes Geschäftsmodell? Vgl. dazu Lobigs/Nordheim (2014) und die Beiträge in diesem Band.

9 Die Begriffe sind zahllos: „Wiki Democracy“, „Open Government“, „Counter Democracy“, „Honeybee Democracy“, „Schwarmdemokratie“, „Liquid-Feedback-System", "Open Data“.

10 So wird von einem sich etablierenden „Überwachungskapitalismus" (Zuboff 2018) gesprochen. 


\section{Aufsätze}

bislang nicht, auszumachen. So war und ist der Vertrauenswert der neuen Intermediäre bei den Nutzenden sehr gering, doch ändert dies nichts an der Nutzung der Dienste.

In der EU, im Europarat und in vielen europäischen Staaten wie aber auch in politischen Gremien der USA hat eine Debatte über diese Phänomene wie über mögliche Regulierungen begonnen (vgl. zum Konzept Media Governance: Puppis 2010). Doch weit ist man nicht, und autoritäre Regime wie China können kein Vorbild sein. ${ }^{11}$ Die globale ökonomische wie technologische Macht sehr weniger Plattformunternehmen - sei es Google, Facebook, Amazon oder Alibaba - ist groß (vgl. Barwise/Watkins 2018). Sie verfügen aber über mehr als nur ökonomische Macht: es kommt die infrastrukturelle, die logistische, die technische (Hard- wie Software), die datenbezogene wie die normenwie regelsetzende Macht dieser privaten Unternehmen hinzu (vgl. Lobigs/Neuberger 2018: 69; vgl. auch Dolata 2018: 123-126). Mit dem Hinweis auf das übergreifende Machtpotential wird deutlich: Es handelt sich um einen Prozess der fundamentalen Institutionalisierung. Die neuen Intermediäre haben normbildende, regelsetzende wie prozedurale Macht (vgl. Jarren 2018a). Mit ihren Allgemeinen Geschäftsbedingungen (AGB) werden durch Private Nutzungs- wie Kommunikationsformen mit Relevanz für die gesellschaftliche Kommunikation normiert. Wer nutzen will, der muss den AGB zustimmen, und die Software definiert, $o b$, was und wie man kommunikativ agieren darf.

Bezogen auf die gesellschaftliche Kommunikation heißt das: Private Akteure konstituieren und regeln individuelle und damit auch die gesellschaftlichen Informations- und Kommunikationsmöglichkeiten wie -formen. Während man dies beispielsweise bei der Einführung des privaten Rundfunks für ein Problem hielt und deshalb spezielle rechtliche Normen wie Gesetze erließ, ist das bezogen auf die neuen Intermediäre (bislang) in den meisten Nationalstaaten unterblieben. ${ }^{12}$ Damit wird zugelassen, dass die neuen Intermediäre nur die allgemeinen nationalstaatlichen rechtlichen Regelungen zu beachten haben, aber keinen medienrechtlichen Vorgaben unterliegen und damit auch keine Rücksicht auf kulturelle Belange nehmen müssen. Sie sind damit auch nicht auf die Wahrnehmung einer öffentlichen Aufgabe verpflichtet. Social Media aber wirken, mehr als es bspw. der private Rundfunk je tat, direkt wie indirekt auf die Öffentliche Meinung ein, und sie gestal-

\footnotetext{
11 In China soll bis 2020 ein Social Credit Score eingeführt werden, mit dem das Verhalten eines Bürgers in einem zentralen Punktekonto erfasst werden könnte.

12 Der Einführung des privaten Rundfunks gingen in Deutschland sogar Versuchsregelungen in Form der sogenannten Kabelpilotprojekte voraus, und es wurde zudem eine spezifische Rundfunkordnung, unter aktiver Beteiligung des deutschen Bundesverfassungsgerichts, nämlich die sog. „duale Rundfunkordnung", mit eigenen Aufsichtsorganen institutionalisiert. Aufgrund der Nichtdefiniton von Social Media als „Medien“ ergeben sich nun besondere Herausforderungen für den Gesetzgeber wie für die Rechtsprechung.
}

ten - auch die nationalstaatliche - Öffentlichkeit mit aus, und zwar strukturell (Vermittlungssystem) wie prozedural (Artikulation wie Aggregation von Meinungen). Sie tun dies nicht wie Massenmedien, also im Sinne der Wahrnehmung eines öffentlichen Auftrages und somit bewusst und intentional mittels des Journalismus, sondern sie wirken „nur" als neutrale Plattformen. Sie sehen sich als Ermöglicher. Sie wollen keine Medien sein. Sie institutionalisieren - zumindest: bislang - keinen Journalismus. Sie erscheinen selbst meinungslos, und sie betonen sogar ihre Meinungs- und somit Interessenlosigkeit. Sie wollen lediglich Besorgungsdienstleister sein. Aber sie haben Einfluss und Macht, auch auf Meinungs- wie Willensbildungsprozesse, so auf die Thematisierung (vgl. Stark/Magin/Jürgens 2017; Neuberger 2018). Sie definieren die neu entstehenden Bereitstellungs- wie Kommunikationsregeln, sie steuern damit soziales Verhalten. Sie erzeugen zum einen direkte, empirisch nachweisbare Wirkungen, so bei Nutzern wie Rezipienten (Mikro-Ebene): Von der Form der Nutzung bestimmter Inhalte bis hin zu „shit storm“ oder „hate speech“. Durch die Bündelung, Entbündelung oder Personalisierung von Informationen und deren Weiterleitung wie Bewertung wird Aufmerksamkeit geschaffen und Nutzung beeinflusst. Durch Aggregation, Selektion wie Präsentation lenken sie Publika wie Nutzerströme - zu Werbe- oder Politikinhalten. Sie lassen das durch ihre Nutzer sammeln und bereitstellen, was die Massenmedien erstellt oder Einzelne selbst produziert und mit anderen geteilt im Netz zur Verfügung gestellt oder hinterlassen haben. Sie lassen: Sie selbst agieren scheinbar nicht. Doch sie haben Einfluss. ${ }^{13}$

Die Intermediäre als nützliche Helfer des Alltags beeinflussen die individuelle wie kollektive Meinungsbildung zudem auf indirekte Weise (vgl. dazu Stark/Magin/Jürgens 2017). Ganz trivial: Sie kosten Zeit, die an anderer Stelle fehlt. Nicht trivial: Sie ziehen Aufmerksamkeit wie Nutzungszeit auf sich und reduzieren die Zuwendungschancen für publizistische Angebote (vgl. Schmidt et al. 2017: 20). Der Zugang zu Nachrichten wie deren Nutzung erfolgt mehr und mehr über Plattformen wie Suchmaschinen (vgl. Stark/Dörr/Aufenanger 2014). Deshalb macht die Produktion wie Bereitstellung eines publizistischen Gesamtangebots ökonomisch keinen Sinn mehr. Informationen wie Wissen werden in anderer Weise er- und verarbeitet, bereitgestellt wie distribuiert. Produktion wie Rezeption erfolgen als fragmentierte Prozesse. Das hat ökonomische Folgen, so bezogen auf die Finanzierungsbereitschaft von (journalistischen) Inhalten, deren Nutzungs- wie Bezahlungswahrscheinlichkeiten unsicher werden.

\footnotetext{
13 Im jüngsten Medien- und Kommunikationsbericht der deutschen Bundesregierung werden die Probleme benannt. Ein politisches Konzept ist allerdings noch nicht auszumachen. Vgl. Die Bundesregierung (2019): Medien- und Kommunikationsbericht der Bundesregierung. Berlin.
} 
Überdies lösen die Intermediäre strukturelle Veränderungen aus: Sie haben Macht, indem sie die Kommunikation von Organisationen beeinflussen, die ihre Mitglieder oder Kunden nun via Social Media erreichen müssen (Meso-Ebene). Und sie haben gesellschaftlichen Impact, weil es sich bei den neuen Intermediären um Institutionen handelt (MakroEbene), die die gesellschaftlichen Erwartungsstrukturen ebenso beeinflussen wie die Legitimität von Angeboten, Kommunikationsformen, Geschäftsmodellen wie Preisen.

\section{Der Institutionalisierungsprozess von Social Media als wirtschafts-, sozial- wie rechtswissenschaftliche Forschungsauf- gabe: Allgemeine Aspekte}

Durch die Ausführungen soll deutlich werden, dass es eine Vielzahl an Phänomenen gibt, an denen der laufende Institutionalisierungsprozess auf der Mikro-, Meso- wie MakroEbene ablesbar ist. Behauptet wird, dass es sich um den Prozess einer fundamentalen - wie aber zugleich auch deshalb spannungsreichen wie partiell widersprüchlichen - Institutionalisierung handelt, der von den neuen Intermediären aktiv vorangetrieben wird. Dieser Prozess bedarf der theoretischen Reflexion wie der empirischen Analyse.

Insbesondere hier sind interdisziplinäre sozialwissenschaftliche Ansätze und Forschungsanstrengungen unverzichtbar, wobei vor allem eine intensivere Kooperation zwischen Wirtschafts- und Kommunikationswissenschaft angezeigt ist: Da die relevanten Social Media-Plattformen Konzernen zugehören, die zutiefst kapitalistisch organisiert und orientiert sind, trifft die aus der Medienökonomie altbekannte Medien-Dichotomie zwischen Wirtschafts- und Kulturgut auch für die Social Media in einer fundamentalen und höchst relevanten Form zu. Noch mehr als bereits in der Ära der klassischen Massenmedien sollte die Medienökonomie somit auch in der digitalen Ära der Plattform-Ökonomie eine Brückenfunktion zwischen den beiden Disziplinen erfüllen.

Gefragt wäre mithin eine Medien- und Internetökonomie, die - im Sinne der Grundlegung von Kiefer (2001) - wirtschaftswissenschaftliche Methoden, Modellierungen und Analysen auf kommunikationswissenschaftliche Problem- und Fragestellungen anwendet. ${ }^{14}$ Mit Blick auf die Analyse des Institutionalisierungsprozesses der neuen Intermediärs-Plattformen können dabei institutionen-, organisations- und verfügungsrechtsökonomische Analysen - im Sinne des auf das Nobelpreisträger-Trio Ronald Coase, Douglass North und Oliver Williamson zurückgehenden modernen institutionentheoretischen Forschungsparadigmas - aufschlussreiche Impulse und Perspektiven generieren (vgl. etwa Lobigs 2018b,c).

14 Dass solche Modellierungen und empirische Analysen hohe relevante Erkenntnispotenziale aufweisen, zeigen aktuell etwa die Studien von Hindman (2018).
Im Hinblick auf die analytische Beschreibung und Erklärung kommunikativ bzw. diskursiv fundierter Formen der Institutionalisierung sind indes auch institutionenökonomische Ansätze augenfällig nicht geeignet. Dies ist die Domäne der Kommunikationswissenschaft, die sich hier auf Ansätze des soziologischen Institutionalismus beziehen kann.

Gesicherte Erkenntnisse, die sich aus medienökonomischen Analysen ergeben, sind dabei freilich als Rahmenbedingungen einer realistischen - und in ihren impliziten normativen Erwartungen nicht überschießenden - Betrachtung wahrzunehmen und zu berücksichtigen, wie dies mit umgekehrten Vorzeichen allerdings auch für die (institutionen-)ökonomische Perspektive gelten sollte. Schon alleine für eine solche wechselseitige Wahrnehmung von Perspektiven und Erkenntnissen bedarf es freilich der wissenschaftspolitischen Unterstützung entsprechender interdisziplinärer Forschungsorganisationen oder der Unterstützung durch Förderstiftungen.

Bevor im Weiteren der Fokus auf die kommunikationswissenschaftliche institutionalistische Analyseperspektive gelegt wird, soll zuvor in Bezug auf das allgemeine interdisziplinäre Feld auf einige allgemeine Forschungsthemen und -fragen hingewiesen werden:

\section{Entrepreneur-Pioniere}

Hinter den Social Media-Plattformen stehen sehr wenige, global bekannte wie global agierende Entrepreneur-Persönlichkeiten, die als technische wie ökonomische Pioniere agieren und so anerkannt sind. Sie vertreten zudem bestimmte neue gesellschaftliche Leitbilder bzw. eine ähnliche „Philosophie“ (Silicon Valley). Sie personifizieren das Angebot, die Organisation wie die Führung und stehen für Innovation, Erfolg. Die Personifizierung gibt den Unternehmen spezifische ökonomische, politische wie soziale Handlungsräume (Gründungsmythos nach dem Motto Garage: aus kleinen Verhältnissen, trotz Abbruch des Studiums u. a. m.), könnte diese aber auch begrenzen.

\section{Etablierung eines organisationalen Feldes}

Innerhalb kurzer Zeit hat sich im Bereich Social Media ein global existierendes organisationales Feld ausgebildet. Interessant für eine Analyse sind die bestehenden Kooperations- wie Konkurrenzverhältnisse. Aus ökonomischer Perspektive stellen sich hier insbesondere Fragen nach Marktmacht und Marktmachtmissbrauch (vgl. etwa Haucap/Stühmeier 2017; Coyle 2018). Zudem ist beachtlich, dass sich bislang in dem Feld noch keine nennenswerten verbände ausgebildet haben. 


\section{Aufsätze}

Etablierung eines relativ einheitlichen wie zugleich unspezifischen Deutungsmusters im organisationalen Feld

Im besagen Feld existiert kein homogenes Deutungsmuster, wohl aber gibt es Gemeinsamkeiten:

- Zugang für jeden,

- einfache Nutzung,

- keine Mitgliedschaften,

- keine direkten monetären Nutzungsgebühren,

- jederzeitige „Austrittsmöglichkeit”.

Aufgrund dieser gemeinsam geteilten Prinzipien hat sich eine gewisse Isomorphie bei den Social Media-Plattformen entwickelt.

\section{Netzwerk und Netzwerkeffekte}

Das "Netzwerk“ ist positiv gesellschaftlich konnotiert. Netzwerke werden aus sozialen Gründen von den Individuen angestrebt. Durch die Betonung der individuellen Nutzerrolle bei den Social Media-Plattformen erhält die Nutzende bzw. der Nutzende eine Wertschätzung (als Individuum). ${ }^{15}$ Und der Wert der Plattform ergibt sich aus der Beteiligung möglichst vieler weiterer Individuen. Der Vorteil für den Einzelnen ist zugleich der Vorteil der Social Media-Plattform als Anbieter: Neben ökonomischen Vorteilen werden zugleich soziale wie kulturelle Legitimitätsvorteile realisiert.

\section{Neuer Organisationstypus}

Plattformen erscheinen nicht als Organisationen, sondern sie bieten als Dienstleistungspartner Inklusionsmöglichkeiten (ohne Mitgliedschaft, ohne Rollen, ohne spezifische Verpflichtungen). Zudem werden die Nutzenden nicht als Kunden oder nur als Nutzende, sondern immer auch als Teil einer „Community“ angesehen und angesprochen. Inklusion wie Exklusion liegt in den Händen der Nutzenden.

\section{Flexible Austauschbeziehungen mit Dritten}

Nicht nur gegenüber den Nutzenden, sondern auch gegenüber Dritten (so bspw. Medienunternehmen) verfügen die Plattformen über flexible, von Außen nicht einfach erkennals auch durchschaubare Kooperations- bzw. Austauschbeziehungen, so wenn Inhalte aus den Massenmedien distribuiert werden. ${ }^{16}$

\footnotetext{
15 Nutzer wirken als Lobbyisten, siehe die Aktivitäten von Kindern und Jugendlichen 2018 im Kontext von YouTube („Man will uns YouTube wegnehmen“). Vgl. dazu grundsätzlich Lobe 2013.

16 Vgl. zum (schwierigen) Verhältnis zwischen den Social Media-Plattformen und journalistischen Medienanbietern etwa Bell (2018).
}

\section{Institutionalisierung neuer elementarer Konfliktlinien}

Den Social Media-Akteuren ist es gelungen, zum Teil im globalen Maßstab, Konfliktlinien aufzubauen bzw. entstehen zu lassen, so bspw. im Medien- oder im Urheberrecht. Ein maßgeblicher Erfolg in der Veränderung von Regelungs- und somit Konfliktlinien liegt u. a. in der von den Akteuren vertretenen Position, keine „Medien“ zu sein. Somit werden keine medienrechtlichen Normen der Nationalstaaten anerkannt. ${ }^{17}$

\section{Fähigkeit zur eigenen Kontraktgestaltung wie zur Neuschöpfung von Rechten}

Die Nutzung wie Benutzung von Social Media-Plattformen basiert auf deren Allgemeinen Geschäftsbedingungen (AGB), die jeweils dem Sitzland des Anbieters entsprechen. Da die Nutzerinnen und Nutzer innen das generelle Recht übertragen müssen, beliebig viele neue Nutzungsrechte an inren Daten schaffen zu können, sind die Plattformen in der Lage, auch gesellschaftlich und politisch höchst wirksame Daten- und Beeinflussungs-Verfügungsrechte kontinuierlich neu zu schaffen (vgl. Lobigs 2018b: 11,16; 2018c).

\section{Datafizierung als Legitimationsstrategie}

Durch die Bereitstellung von Daten für Individuen wie für andere wird eine Form der „objektiven“ Möglichkeit für eine „sichere" wie „korrekte" Orientierung geschaffen. Dadurch wird der Einfluss anderer Datenerzeuger wie Datenverwender relativiert, zumal jener, die nicht in vergleichbarer Form Daten bereitstellen können. Das wirkt als Entmystifizierung bisheriger gesellschaftlicher Autoritäten wie Experten, die datenbasiert argumentieren (vgl. hierzu grundlegend Mau 2017).

\section{Der Institutionalisierungsprozess von Social Media als kommunikationswissen- schaftliche Forschungsaufgabe}

Im Institutionalisierungsprozess der Social Media-Plattformen etablieren sich neue, fundamentale Institutionen - und dies geschieht wesentlich kommunikativ (institutional work). Die Institutionalisierung vollzieht sich global wie dezentral und unter Beteiligung der Gesellschaftsmitglieder. Teilhabe- wie Teilnahmemöglichkeiten gehen über das, was die Massenmedien bieten, dabei weit hinaus.

Beim Prozess der Etablierung von Social Media-Plattformen handelt es sich - so die hier vertretene Annahme - im Kern also um eine Form der kommunikativen Institutionalisierung (vgl. Cornelissen et al. 2015; Sandhu 2018). Kommunikativ meint, dass nicht, wie bis anhin üblich, politisch-staatliche Akteure eine Rahmenordnung definieren und sodann recht-

17 Vgl. hierzu kritisch etwa Milborn/Breitenecker (2018: insb. 89-118). 
liche Instanzen die Institutionalisierung maßgeblich prägen, sondern private Unternehmen gestalten unter Mitwirkung der individuellen Nutzer eine Kommunikationsordnung im Prozess aus (vgl. dazu Jarren 2018b): Auf der einen Seite stehen die Anbieter mit ihrer starken Werbe-, Marketingwie PR-Macht. Mittels Formen der persuasiven Kommunikation werden Nutzer gewonnen, wird systematisch die erzeugte Intransparenz - etwa über algorithmische Entscheidungsverfahren wie den Umgang mit Daten - zu überspielen versucht. Auf der anderen Seite stehen die Nutzenden, die die innen gebotenen Kommunikationsmöglichkeiten in Anspruch nehmen - und die dadurch soziale Macht (kommunikativen Einfluss) als Option erlangen. ${ }^{18}$

Aus diesen Überlegungen ergibt sich, dass die wesentlichen Aspekte des kommunikativen Institutionalisierungsprozesses vor allem aus einer kommunikationswissenschaftlichen Perspektive analysiert werden sollten. Für ein solches Forschungsprogramm wären beispielsweise folgende Aspekte von Relevanz:

\section{Globale Konfliktlinien und neue Formen der kommunikativen Sichtbarkeit}

Im Kontext mit den Nutzungsmöglichkeiten wie -einschränkungen bei den neuen Intermediären werden unterschiedliche politische Regime kommunikativ sichtbar gemacht. Die Rechte oder die Optionen von Einzelnen gegenüber den politischen Machtstrukturen werden sichtbar. Konflikte um die Informations- wie Kommunikationsfreiheit werden in den Zusammenhang mit den Plattformen gebracht, so durch die Berichterstattung in den Massenmedien.

\section{Etablierung eines globalen Leitbild-Feldes}

Kommunikativ wird von den Social Media-Plattformen eine Art von weltgesellschaftlicher Gemeinschaft als Leitbild behauptet („Community“). Mit dieser Grundidee wird auf Kooperation und Kollaboration gesetzt. Nutzerinnen und Nutzer sind keine Kunden, sie sind Teil einer (globalen) Gemeinschaft.

\section{Transzendentale Begriffe als kommunikative Hebel}

Social Media-Plattformen streben „Community“ an, aber darüber hinaus bedienen sich die Akteure einer Reihe von Begriffen, mit denen auf weitere Gesellschafts- oder Gemeinschaftsverständnisse bzw. gesellschaftliche Ordnungsverhältnisse Bezug genommen wird. Beispiele: open, connected, shared, liquid, modular, co-creation, crowds,

18 Für mögliche Regulierungsansätze wären demnach kommunikative Maßnahmen relevant. Diese Möglichkeit sollte vertieft geprüft werden. Siehe dazu erste Überlegungen bei Jarren (2018b, 2019). informal u. a. m. Hiermit wird zwar ein genereller Wandel angesprochen, gleichzeitig wird aber auch auf traditionelle gesellschaftliche Vorstellungen Bezug genommen.

\section{Interessenkommunikation der Intermediäre als institutionelle Legitimationshandlung}

Die Nutzerinnen und Nutzer werden persönlich angesprochen, als Teil einer "Community“. Die Interessen der Plattformen werden institutionell definiert („Netzwerk“, „Community") und in die kommunikativ bei den Nutzerinnen und Nutzern erzeugten Erwartungen eingebettet. Dabei wird aber überwiegend an den einzelnen Nutzer (als Teil einer Gemeinschaft wie der Plattform) adressiert kommuniziert. „Wir": Anbieter und Nutzer sind eine Gemeinschaft („Teil sein“). Zugleich aber stehen den Nutzerinnen und Nutzern auf Seiten der Plattformen weder persönliche MailAnschriften noch konkrete Personen als Ansprechpersonen zur Verfügung. Die Kommunikationsmöglichkeiten zwischen den Nutzern und den Plattformen sind hochgradig begrenzt. So kann man empirisch sehen, dass die mit der Nutzung verbundene Unsicherheitsabsorption im Kern nicht mit Hilfe von Maßnahmen der Plattformen erfolgt, sondern durch die Kommunikation innerhalb der Nutzergruppen erfolgt oder erfolgen muss. ${ }^{19}$

\section{Institutionelle Legitimität als spezifisches Kommunikationsmodell}

Die institutionelle Legitimität der Social Media-Plattformen und ihres Geschäftsmodells basiert auf unterschiedlichen kommunikativen Prozessen. Sie ergibt sich aber wesentlich nicht aus den Nutzern zugewiesenen Rollen zur Mitwirkung an der Plattform, sondern vorrangig im Versprechen der Plattform an die Nutzer, sich

- einerseits selbst ein Netzwerk kommunikativ aufbauen und gestalten zu können und

- andererseits mittels der Benutzungsmöglichkeit der Plattform, allein oder mit anderen, kommunikativen Einfluss oder sogar kommunikative Macht zu erlangen.

Social Media beinhalten für Einzelne (exemplarisch: die sog. „Influencer"), Netzwerke, Gruppen wie Organisationen das Versprechen der wirksamen, der auch öffentlich wirksamen, Kommunikation. Diese Option verleiht den Plattformen institutionelle Autorität, weil sie ihren Nutzern materielle Vorteile (so dem Influencer) oder zumindest kommunikative Autorität suggeriert (und partiell auch gewährt).

\footnotetext{
19 Eine eigentliche Nutzer- wie auch Verbraucher- bzw. Konsumentenberatung, auch durch Dritte, existiert nur im sehr geringen Maße. Die Forderungen nach - mehr - Medienkompetenz aber sind hoch.
} 


\section{Aufsätze}

\section{Institutionelle Legitimität durch staatliche wie öffentliche Sichtbarkeit wie Anerkennung}

Staatliche Instanzen, Amtsinhaber, politische Akteure auf allen Stufen des politischen Systems, aber auch öffentliche Einrichtungen wie Unternehmen, sind auf Social Media aktiv. Sie schaffen damit eine allgemeine Sichtbarkeit für die Plattformen und diese erhalten dadurch einen öffentlichen - möglicherweise sogar einen quasi-amtlichen - Charakter. Vor allem wenn hoheitlich wirkende Organisationen, wie Polizeibehörden, sich der Social Media bedienen, kommt dies einer Art von Prüfsiegel gleich..$^{20}$

\section{Schlussbemerkungen}

Im Beitrag wurden Aspekte genannt, die - um es mit Kiefer/ Steiniger (2014: 77 f.) zu formulieren - einer fundamentaIen Institutionalisierung entsprechen. Kennzeichen fundamentaler Institutionen sind:

- Sie grenzen Handlungs- wie Gestaltungspielräume auf den nachfolgenden gesellschaftlichen Ebenen ein.

- Sie bilden sich zumeist in einem evolutionären Prozess aus.

- Sie legen Handlungsrechte und Handlungspflichten fest.

- Sie werden zumeist unbewusst verfolgt und dabei nicht hinterfragt.

- Sie verfügen über eine starke soziale Bindungskraft, aber ohne über gesetzliche Regeln oder Strafmöglichkeiten selbst zu verfügen.

- Sie verbinden zumeist eine Gemeinschaft, so durch die Verfolgung bestimmter allgemeiner Ziele.

Ziel der Forschung sollte es sein, zu prüfen, ob Social Media-Plattformen dieses Macht- bzw. Einflusspotential zugeschrieben werden kann. Hier sind vor allem auch wirtschafts- wie rechtswissenschaftliche Beiträge notwendig.

Institutionalisierungsprozesse können als Kommunikationsprozesse aufgefasst und entsprechend theoretisch gefasst wie empirisch analysiert werden. Die «zuständige» Disziplin ist an dieser Stelle die Kommunikationswissenschaft, die dabei freilich die ökonomischen und juristischen Herausforderungen und Rahmenbedingungen berücksichtigen muss, die die Gegenstände medienökonomischer und medien-

20 Vor dem Hintergrund dieser Tatsache mutet es seltsam an, wenn politische Akteure, die im hohen Maße Social Media nutzen und benutzen, im Falle von informationstechnischen Defiziten zu ihren Lasten - wie im jüngsten Fall im Januar 2019 in Deutschland - sich öffentlich über bereits bekannte Probleme wie Risiken aufregen. Die skandalisierten Vorgänge wären ein Anlass gewesen, die eigene Beteiligung zu prüfen sowie zu klären, ob staatliche Stellen oder öffentliche Einrichtungen aktiv mitwirken können bzw. dürfen im Wissen um datenschutzrechtliche wie datenschutztechnische Mängel bei Plattformen. Durch diese Beteiligung üben öffentliche Einrichtungen einen Nutzerzwang aus rechtlicher Untersuchungen sind. Auch hier ist somit die Notwendigkeit einer intensiven interdisziplinären Verständigung und Zusammenarbeit gegeben.

Dabei wäre zu klären, welche Bedeutung Kommunikation für dauerhafte Institutionalisierungen zuerkannt werden kann. Ist Kommunikation eine starke oder schwache Ressource? Trotz der Ausbildung eines organisationalen Feldes und trotz ökonomisch starker Social Media-Plattformen stellt sich die Frage, ob sich die neuen Anbieter dauerhaft erfolgreich institutionalisieren können. Wie man sieht, kann mittels Kommunikation erfolgreich institutional work betrieben werden. Zugleich aber sind mit den Social MediaPlattformen für die Nutzenden gelingende wie erfolgreiche, also auch wirkungsstarke, Kommunikationserwartungen verbunden. Können diese Erwartungen von den Plattformen, die sich in einem Konkurrenzverhältnis befinden und als Plattformen auf große Nutzergemeinschaften setzen müssen, dauerhaft erfüllt werden? Zudem können die den Plattformen unterliegenden „Philosophien“ sowie zugrundliegenden Leitideen kommunikativ in Frage gestellt werden: Einerseits durch Akteure sowie politische Diskurse in der Gesellschaft. Und andererseits auch durch die Plattformen selbst, so im Falle der Dominanz ökonomischer Kurzeitinteressen oder offenkundiger Verstöße gegen selbstdefinierte Ziele („Wir schützen Eure Daten“), die zu einem grundlegenden Vertrauensproblem werden könnten.

Die Klärung der Institutionalisierungspotenziale der Ressource Kommunikation ist der zentrale Beitrag, den die Kommunikationswissenschaft in der interdisziplinären Untersuchung des Institutionalisierungsprozesses der Social Media leisten kann und muss. Technologische, ökonomische und juristische Analysen sind indessen notwendig, um das Verständnis der Kräfte- und Spannungsfelder zu präzisieren, die die Grenzen und Herausforderungen dieser Ressource Kommunikation definieren.

\section{Vier Aspekte seien dabei in Form von Fragen abschließend hervorgehoben:}

1. Social Media-Plattformen geben zwar ihren Nutzerinnen und Nutzern ein prinzipielles Inklusions- wie Beteiligungsversprechen, sie selbst aber bieten den Nutzern keine organisationale Teilhabe oder gar Mitwirkung - obwohl sie grundsätzlich für Partizipation stehen. Durch das Handeln der Nutzerinnen und Nutzer entstehen Netzwerke wie (Sub-)Gruppen, die jedoch gegenüber der Plattform, so auch bezogen auf benötigte spezifische Dienstleistungen, nicht handlungsfähig sind. Einzelne wie Gruppen sind somit nur Adressaten von Kommunikation - und das meint: persuasiver, hierarchischer Kommunikation - durch die Betreiber der Plattformen. Kann dies vor dem Hin- 
tergrund der vertretenen und kommunikativ stark betonten partizipativen Leitideen der Plattformen deren institutionelle Legitimität sichern?

2. Durch die Nichthandlungsfähigkeit der Nutzenden kann die Dienstleistungs- oder Besorgungsleistung möglicherwiese eingeschränkt sein. Dies kann im Widerspruch zum personalisierten Dienstleitungsanspruch gebracht werden und die Grenzen großer Netzwerke aufzeigen („Massennetzwerk“; „Pseudoindividualisierung“; „, industriell anmutende Konfektionalisierung"). Wird das Prinzip des offenen Netzwerkes, in das sich jederzeit jemand selbst inkludieren und sodann vielfältig kollaborieren kann, zum Problem?

3. Offensichtlich ist, dass Unsicherheiten bei der Institutionalisierung kommunikativ (persuasive Kommunikation: PR, Werbung, Marketing, Lobbying) gesteuert, beeinflusst wie abzufangen versucht werden. Kann sich aber die „eingeschriebene Institution" der „Thematisierung als strittige Institution" (Katzenbach 2018: 327) entziehen? Was sind die Bedingungen für institutionelles Vertrauen bei den neuen Intermediären?

4. Social Media-Plattformen beeinflussen die Massenmedien wie den Journalismus. Doch bedarf eine Institution Social Media zu ihrer Legitimation nicht einer allgemeinen - und nicht allein von innen konstituierten - Öffentlichkeit?

Der Institutionalisierungsprozess der Social Media ist eine spannende Herausforderung für die Forschung, weil dieser Prozess nur in Kooperation zwischen unterschiedlichen Disziplinen betrieben werden kann. Natürlich muss empirisch gearbeitet werden. Doch bedarf es zugleich einer Intensivierung der theoretischen Bemühungen.

\section{Literatur}

Barwise, P./Watkins, L. (2018): The Evolution of Digital Dominance: How and Why We Got to GAFA, in: Moore, M./Tambini, D. (Hrsg.): Digital Dominance: The Power of Google, Amazon, Facebook, and Apple. Oxford, S. 21-49.

Bell, E. (2018): The Dependent Press: How Silicon Valley Threatens Independent Journalism. in: Moore, M./Tambini, D. (Hrsg.): Digital Dominance: The Power of Google, Amazon, Facebook, and Apple. Oxford, S. 241-261.

Berger, P. L./Luckmann, T. (2007): Die gesellschaftliche Konstruktion der Wirklichkeit, 21. Auflage, Frankfurt am Main.

Bründl, S./Matt, C./Hess, T. (2016): Daten als Geschäft - Rollen und Wertschöpfungsstrukturen im deutschen Markt für persönliche Daten, in: Wirtschaftsinformatik \& Management, 6, S. 78-83.

Cornelissen, J. P./Durand, R./Fiss, P. C./Lammers, J. C./Naara, E. (2015): Putting Communication Front and Center in Institutional Theory and Analysis, in: Academy of Management Review, 40 (1), S. 10-27.

Coyle, D. (2018): Platform Dominance: The Shortcomings of Antitrust Policy, in: Moore, M./Tambini, D. (Hrsg.): Digital Dominance: The Power of Google, Amazon, Facebook, and Apple, Oxford, S. 50-70.

Di Fabio, U. (2016): Grundrechtsgeltung in digitalen Systemen: Selbstbestimmung und Wettbewerb im Netz, Studie im Auftrag der VG Media, München.
Die Bundesregierung (2019): Medien- und Kommunikationsbericht der Bundesregierung 2018, Berlin. https://www.bundesregierung.de/resource/blob/975226/1567556/2ccf0dfef063d7b16e8c24fa94f39d70/2019-01-09-medienbericht-breg-2018-politischer-teil-data.pdf?download=1

Dolata, U. (2018): Internetkonzerne: Konzentration, Konkurrenz und Macht, in: Dolata, U./Schrape, J.-F. (Hrsg.): Kollektivität und Macht im Internet: Soziale Bewegungen - Open Source Communities - Internetkonzerne, Wiesbaden, S. $101-130$

Donges, P. (2006): Medien als Institutionen und ihre Auswirkungen auf Organisationen: Perspektiven des soziologischen Neo-Institutionalismus für die Kommunikationswissenschaft, in: Medien \& Kommunikationswissenschaft, 54. Jg., H. 4, S. 563-578.

Evans, D. S./Schmalensee, R. (2016): Matchmakers: The New Economics of Multisided Platforms, Boston.

Gillespie, T. (2018): Regulation of and by Platforms, in: Burges, J./Marwick, A./Poell, T. (Hrsg.): The SAGE Handbook of Social Media, London, S. 254278. http://dx.doi.org/10.4135/9781473984066.n15

Gläser, M./Gounalakis, G./Hess, T./Lobigs, F./Neuberger, C./Sjurts, I. (2018): Inhaltliche Neupositionierung der MedienWirtschaft. In: MedienWirtschaft, 15 Jg., H. 4, S. 4-5.

Haucap, J./Stühmeier, T. (2016): Competition and Antitrust in Internet Markets, in: Bauer, J. M./Latzer, M. (Hrsg.): Handbook on the Economics of the Internet, Cheltenham u. a., S. 183-210.

Hindman, M. (2018): The Internet Trap: How the Digital Economy Builds Monopolies and Undermines Democracy, Princeton/Oxford.

Jarren, 0. (2019): Verantwortungskultur in der Kommunikationsgesellschaft: Kommunikationspolitik als Ansatz zur Ausgestaltung der digitalen Medienwelt, in: Seibert-Fohr, A. (Hrsq.): Verantwortung in entgrenzten Räumen, Wiesbaden (Titel für Band noch vorläufig) (Manuskript).

Jarren, 0. (2018a): Normenbildende Macht: Intermediäre als gesellschaftliche Herausforderung, in: epd Medien, 25, 15. Juni 2018, S. 35-39.

Jarren, 0. (2018b): Kommunikationspolitik für die Kommunikationsgesellschaft: Verantwortungskultur durch Regulierung, in: Aus Politik und Zeitgeschichte, $40-41,1.10 .2018$, S. 23-28.

Jarren, 0. (2016): Nicht Daten, sondern Institutionen fordern die Publizistik- und Kommunikationswissenschaft heraus: Zu Andreas Hepps Beitrag „Kommunikations- und Medienwissenschaft in datengetrieben Zeiten", in: Publizistik, 61. Jg., H. 4, S. 373-383

Jarren, 0. (2008): Massenmedien als Intermediäre: Zur anhaltenden Relevanz der Massenmedien für die öffentliche Kommunikation, in: Medien \& Kommunikationswissenschaft, 56. Jg. H. 3-4, S. 329-346.

Katzenbach, C. (2018): Die Regeln digitaler Kommunikation: Governance zwischen Norm, Diskurs und Technik, Wiesbaden.

Kiefer, M. L. (2017): Journalismus als Dienstleistung? Eine dienstleistungstheoretische Einschätzung, in: Medien \& Kommunikationswissenschaft, 65. Jg. H. 4, S. 682-703.

Kiefer, M. L. (2001): Medienökonomik, 1. Aufl., München/Wien.

Kiefer, M. L./Steininger, C. (2014): Medienökonomik, 3. Aufl., München.

Lobe, A. (2013): Nutzer sind die besten Lobbyisten, in: Frankfurter Allgemeine Zeitung, 4.11.2013, S. 13.

Lobigs, F. (2017): Paradigmenwechsel in der Ökonomie gesellschaftlich relevanter digitaler Medieninhalte. Expertise im Auftrag der Schweizer Eidgenössischen Medienkommission EMEK. (Präsentation und Veröffentlichung am 24.4.2017 in Bern. (www.emek.admin.ch/inhalte/pdf/Expertise_EMEK Frank Lobigs_v.pdf).

Lobigs, F. (2018a): Wirtschaftliche Probleme des Journalismus im Internet: Verdrängungsängste und fehlende Erlösquellen, in: Neuberger, C./Nuernbergk C. (Hrsg.): Journalismus im Internet: Profession - Partizipation - Technisierung, 2. Aufl., Berlin/Heidelberg/Wiesbaden, S. 295-334.

Lobigs, F. (2018b): Zum Verhältnis von ökonomischem und diskursivem Institutionalismus am Beispiel der Plattform-Revolution von Medien und

Öffentlichkeit. Folien eines Vortrags auf dem Workshop Kommunikationswissenschaftliche Perspektiven des Institutionalismus. 16./17.11.2018 am IKMZ Institut für Kommunikationswissenschaft und Medienforschung, Universität Zürich.

Lobigs, F. (2018c): Die Fundamentalzäsur: Digitaler Strukturwandel der Öffentlichkeit und die Funktionsfähigkeit moderner Demokratien: Eine institutionenökonomische Analyse, in: Blum, R./Eisenegger, M./Ettinger, P./Prinzing, M. (Hrsg.): Digitaler Strukturwandel der Öffentlichkeit: Historische Verortung, Modelle und Konsequenzen. Wiesbaden (i. V.).

Lobigs, F./Neuberger, C. (2018): Meinungsmacht im Internet und die Digitalstrategien von Medienunternehmen: Neue Machtverhältnisse trotz expandierender Internet-Geschäfte der traditionellen Massenmedien-Konzerne, Berlin (Gutachten für die Kommission zur Ermittlung der Konzentration im Medienbereich)

Lobigs, F./Nordheim, G. von (2014): Journalismus und Ökonomie - getrennt und doch verbunden, in: Lobigs, F./Nordheim, G. von (Hrsg.): Journalismus ist kein Geschäftsmodell, Baden-Baden, S. 7-16. 


\section{Aufsätze}

Mau, S. (2017): Das metrische Wir: Über die Vermessung des Sozialen, Frankfurt am Main.

Milborn, C./Breitenecker, M. (2018): Change the Game, Wien.

Neuberger, C. (2018): Meinungsmacht im Internet aus kommunikationswissenschaftlicher Sicht, in: UFITA - Archiv für Medienrecht und Medienwissenschaft, Jg. 82, H. 1, S. 53-68.

Puppis, M. (2010): Media Governance: A New Concept for the Analysis of Media Policy and Regulation, in: Communication, Culture and Critique, Vol. 3, Issue 2. S. 134-149. https://doi.org/10.1111/j.1753-9137.2010.01063.x

Sandhu, S. (2018): Kommunikativer Institutionalismus und Accounts: Sprachliche Muster der Legitimation in der Public Relations, in: Schach, A./Christoph, C. (Hrsg.): Handbuch Sprache in den Public Relations, Wiesbaden, S. 21-36.

Schmidt, J.-H./Merten, L./Hasebrink, U./Petrich, I./Rolfs, A. (2017): Zur Relevanz von Online-Intermediären für die Meinungsbildung, Hamburg (Arbeitspapiere des Hans-Bredow-Instituts, 40).

Schrader, M. (2019): Die digitale Transformation steckt fest, in: Frankfurter Allgemeine Zeitung, 31.12.2018, S. 18.

Schulz, W./Dankert, K. (2016): Die Macht der Informationsintermediäre: Erscheinungsformen, Strukturen und Regulierungsoptionen, Bonn.

Stark, B./Magin, M./Jürgens, P. (2017): Ganz meine Meinung? Informationsintermediäre und Meinungsbildung: Eine Mehrmethodenstudie am Beispiel von Facebook, Düsseldorf (Landesanstalt für Medien).

Stark, B./Dörr, D./Aufenanger, S. (Hrsg.)(2014): Die Googleisierung der Informationssuche: Suchmaschinen zwischen Nutzung und Regulierung, Berlin.

Vorderer, P. et al. (2015): Der mediatisierte Lebenswandel: Permanently Online, Permanently Connected, in: Publizistik, 60. Jg., H. 3, S. 259-276.

Zuboff, S. (2018): Das Zeitalter des Überwachungskapitalismus, Frankfurt am Main. 